\title{
Alterations in acylcarnitines, amines, and lipids inform about the mechanism of action of citalopram/escitalopram in major depression
}

Siamak MahmoudianDehkordi', Ahmed T. Ahmed ${ }^{2}$, Sudeepa Bhattacharyya ${ }^{3}$, Xianlin Han ${ }^{4}$, Rebecca A. Baillie (0), Matthias Arnold (1), ${ }^{1,6}$ Michelle K. Skime', Lisa St. John-Williams ${ }^{8}$, M. Arthur Moseley ${ }^{8}$, J. Will Thompson ${ }^{8}$, Gregory Louie 10', Patricio Riva-Posse ${ }^{9}$, W. Edward Craighead (10), William McDonald ${ }^{9}$, Ranga Krishnan ${ }^{10}$,

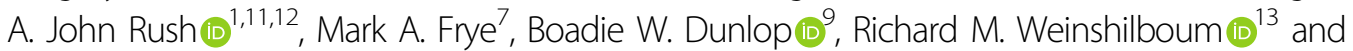
Rima Kaddurah-Daouk $\mathbb{D}^{1,14,15}$, The Mood Disorders Precision Medicine Consortium (MDPMC)

\begin{abstract}
Selective serotonin reuptake inhibitors (SSRIs) are the first-line treatment for major depressive disorder (MDD), yet their mechanisms of action are not fully understood and their therapeutic benefit varies among individuals. We used a targeted metabolomics approach utilizing a panel of 180 metabolites to gain insights into mechanisms of action and response to citalopram/escitalopram. Plasma samples from 136 participants with MDD enrolled into the Mayo Pharmacogenomics Research Network Antidepressant Medication Pharmacogenomic Study (PGRN-AMPS) were profiled at baseline and after 8 weeks of treatment. After treatment, we saw increased levels of short-chain acylcarnitines and decreased levels of medium-chain and long-chain acylcarnitines, suggesting an SSRI effect on $\beta$-oxidation and mitochondrial function. Amines-including arginine, proline, and methionine sulfoxide-were upregulated while serotonin and sarcosine were downregulated, suggesting an SSRI effect on urea cycle, one-carbon metabolism, and serotonin uptake. Eighteen lipids within the phosphatidylcholine (PC aa and ae) classes were upregulated. Changes in several lipid and amine levels correlated with changes in 17-item Hamilton Rating Scale for Depression scores $\left(\mathrm{HRSD}_{17}\right)$. Differences in metabolic profiles at baseline and post-treatment were noted between participants who remitted $\left(\mathrm{HRSD}_{17} \leq 7\right)$ and those who gained no meaningful benefits $\left(<30 \%\right.$ reduction in $\left.\mathrm{HRSD}_{17}\right)$. Remitters exhibited (a) higher baseline levels of C3, C5, alpha-aminoadipic acid, sarcosine, and serotonin; and (b) higher week-8 levels of PC aa C34:1, PC aa C34:2, PC aa C36:2, and PC aa C36:4. These findings suggest that mitochondrial energetics - including acylcarnitine metabolism, transport, and its link to $\beta$-oxidation —and lipid membrane remodeling may play roles in SSRI treatment response.
\end{abstract}

Correspondence: Rima Kaddurah-Daouk (kaddu001@mc.duke.edu) ${ }^{1}$ Department of Psychiatry and Behavioral Sciences, Duke University School of Medicine, Durham, Durham, NC, USA

2Department of Neurology, Mayo Clinic, Rochester, MN, USA

Full list of author information is available at the end of the article

These authors contributed equally: Siamak MahmoudianDehkordi, Ahmed

T. Ahmed.

A list of authors and their affiliations appears at the end of the paper.

\section{Introduction}

Major depressive disorder (MDD) is a common, often disabling condition that affects more than 300 million individuals worldwide ${ }^{1}$, but much about its pathobiology and the biology of treatment response remains unknown. Selective serotonin reuptake inhibitors (SSRIs) are common first-line agents used for the treatment of $\mathrm{MDD}^{2,3}$, yet $\sim 40 \%$ of patients who receive SSRI treatment do not respond and more than two-thirds do not achieve

\section{(c) The Author(s) 2020}

(c) Open Access This article is licensed under a Creative Commons Attribution 4.0 International License, which permits use, sharing, adaptation, distribution and reproduction cc) in any medium or format, as long as you give appropriate credit to the original author(s) and the source, provide a link to the Creative Commons license, and indicate if changes were made. The images or other third party material in this article are included in the article's Creative Commons license, unless indicated otherwise in a credit line to the material. If material is not included in the article's Creative Commons license and your intended use is not permitted by statutory regulation or exceeds the permitted use, you will need to obtain permission directly from the copyright holder. To view a copy of this license, visit http://creativecommons.org/licenses/by/4.0/. 
remission of symptoms ${ }^{4}$. Clinical symptoms are insufficient to guide treatment selection for individual patients ${ }^{5}$. Hence, in clinical practice, a "trial-and-error" approach is used to find an effective therapy ${ }^{6}$.

MDD patients who achieve remission after treatment with an antidepressant appear to have a metabolic state distinct from never-depressed individuals, with alterations in methylation, purine metabolism, and oxidative stress pathways ${ }^{7}$. Pilot studies have implicated metabolic dysregulation in the pathogenesis of MDD, with alterations in several pathways, including neurotransmission (GABA, glutamine, tryptophan, phenylalanine), nitrogen metabolism, methylation, and lipid metabolism ${ }^{8-12}$.

A metabolomics approach provides tools to enable the mapping of global metabolic changes in neuropsychiatric diseases and upon treatment ${ }^{7-9,13-18}$. Pharmacometabolomics - the application of metabolomics to the study of drug effects-has been successfully used to map the effects of sertraline ${ }^{19}$, ketamine, and placebo ${ }^{20}$ by providing new insights about mechanisms of action and response $^{19,21,22}$.

Several studies have shown mitochondrial dysfunction or lower adenosine triphosphate (ATP) production in acutely depressed MDD patients, which suggests a role for mitochondrial energetics in depression ${ }^{23-27}$. Furthermore, accumulated evidence suggests that alterations of acylcarnitines may contribute to several neuropsychiatric diseases, including depression ${ }^{28-32}$, autism ${ }^{33}$, and schizophrenia $^{34,35}$. Acylcarnitines are a class of metabolites that are formed from the transfer of the acyl group of a fatty acyl-Coenzyme A (CoA) to carnitine, which is catalyzed by carnitine acyltransferases in the mitochondria (Fig. 1) ${ }^{36-38}$. Carnitine deficiency or carnitine acyltransferase dysfunction reduces $\beta$-oxidation of fatty acids, and therefore reduced mitochondrial energy (ATP) production. Hence, altered plasma or serum acylcarnitine levels can be used as biomarkers that suggest abnormalities in beta-oxidation (e.g., inborn errors of metabolism $)^{39-43}$. Elevated medium- and long-chain acylcarnitine concentrations in blood have been associated with incomplete $\beta$-oxidation of fatty acids in a rat model of depression $^{44}$.

Changes in the levels of amino acids and biogenic amines such as histidine, kynurenine, methionine sulfoxide, arginine, citrulline, ornithine, and urea have been implicated in $\mathrm{MDD}^{45-53}$. Blood lipid changes have also been implicated in the pathophysiology of depression, schizophrenia, and Alzheimer's disease ${ }^{54-58}$. Lipids are involved in crucial brain functions, including cell membrane structure, membrane transmitters, and regulation of synapses, as well as biological messenger functions, energy metabolism, and neuroendocrine function ${ }^{59}$. MDD entails disturbances in the regulation of the molecular pathways of neurotransmitter systems, synaptic plasticity, and neuroendocrine and immune regulation ${ }^{60}$. Therefore, lipid and amino acid analyses may contribute to finding MDD-relevant biomarkers ${ }^{61}$.

In this study, we used a targeted metabolomics approach to interrogate possible functions for acylcarnitines, amino acids, biogenic amines, and lipids to addresses the following questions regarding mitochondrial function and neurotransmission in MDD:

1. What are the overall changes in the metabolic profile over 8 weeks of SSRI exposure, and which of these metabolic changes are related to each other?

2. Which metabolite-level changes are related to improvements in depressive symptoms (17-item Hamilton Rating Scale for Depression $\left.\left[\mathrm{HRSD}_{17}\right]\right)$ over the 8 weeks of SSRI treatment?

3. Are there baseline metabolites that differentiate between patients who have benefitted substantially $\left(\mathrm{HRSD}_{17} \leq 7\right.$, i.e., "remitters") after 8 weeks of treatment vs. those who gained no meaningful benefits with SSRI treatment $(<30 \%$ reduction in $\mathrm{HRSD}_{17}$ from baseline to week 8 , i.e., "treatment failures")?

4. Are there week 8 metabolites that differentiate between remitters and treatment failures?

\section{Materials and methods}

\section{Study design and participants}

This study examined plasma samples from 136 participants with MDD who were enrolled in the Mayo Pharmacogenetics Research Network Antidepressant Medication Pharmacogenetics Study (PGRN-AMPS) (ClinicalTrials.gov NCT00613470). The design and clinical outcomes of the PGRN-AMPS study have been described in detail elsewhere ${ }^{62}$. The trial enrolled 800 MDD participants 18-84 years of age from Mayo Clinic psychiatry or primary care clinics. Patients who met diagnostic criteria for MDD without psychosis or mania and who had a score of $>14$ on $\mathrm{HRSD}_{17}$ were eligible for inclusion in the trial. Subjects with medical contraindications to citalopram or escitalopram treatment or who had previously failed to respond to an adequate course of citalopram or escitalopram, or who had been on an antipsychotic, or mood-stabilizing medication were excluded. The participants received 8 weeks of open-label treatment with either citalopram $(20-40 \mathrm{mg} /$ day $)$ or escitalopram (10-20 mg/day). MDD severity was assessed using the $\mathrm{HRSD}_{17}{ }^{63}$ at each visit. The PGRN-AMPS protocol was approved by the Mayo Clinic Institutional Review Board. All risks and benefits of the PGRN-AMPS study were discussed with participants, each of whom gave written informed consent prior to entering the study. For this project, we selected those subjects who had a $30 \mu \mathrm{l}$ of aliquot available for baseline and 8-week visits for analysis without further thawing and subaliqouting. There 


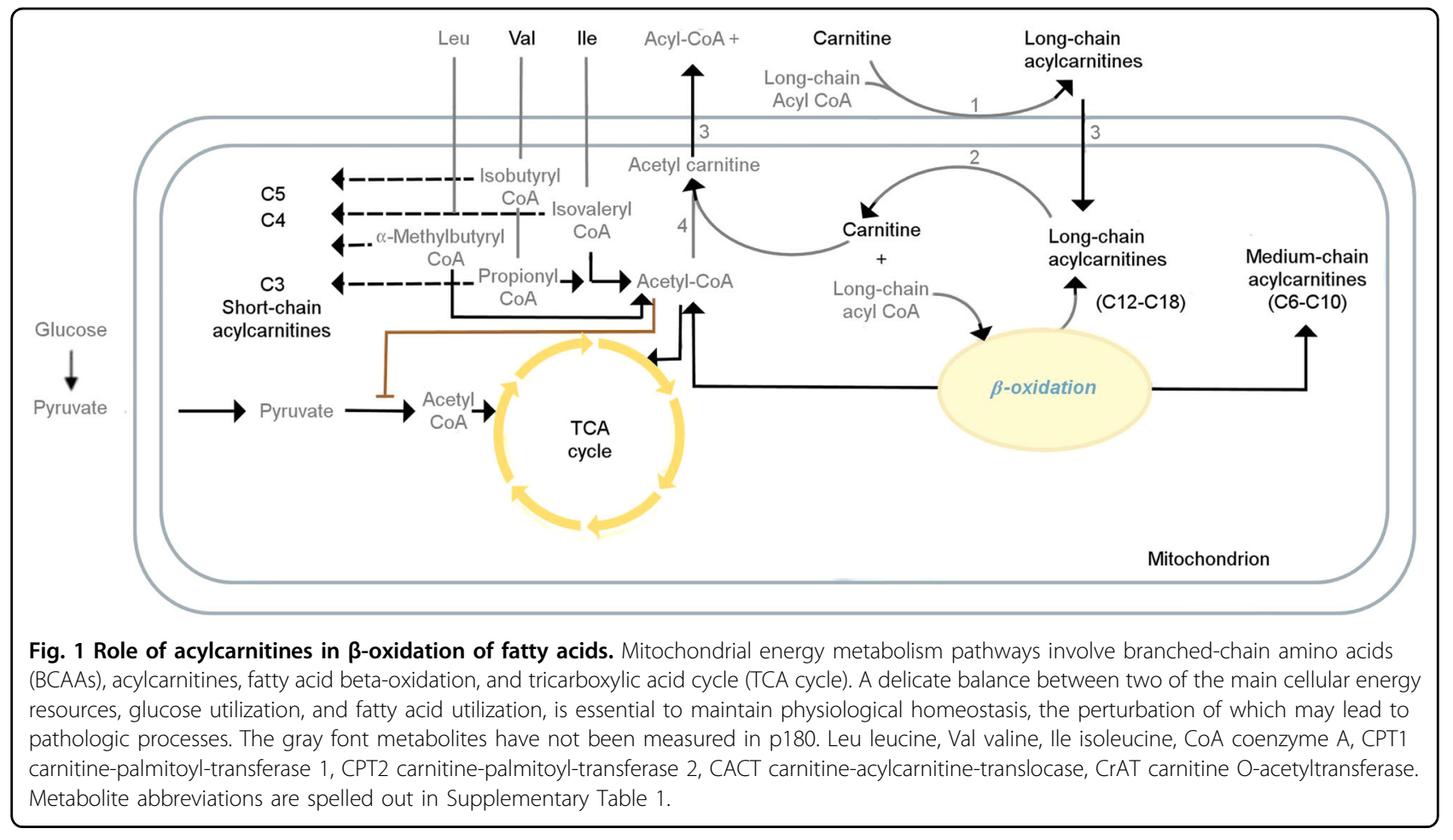

is no placebo group for this project as we were not testing the efficacy of the medication but using the citalopram/ escitalopram as a probe drug to study the contribution of pharmacogenomics in variations of mood outcomes.

\section{Metabolomic profiling using the absolute IDQ p180 Kit}

Using the AbsoluteIDQ ${ }^{\circledR}$ p180 Kit (BIOCRATES Life Science AG, Innsbruck, Austria), we measured metabolites with a targeted metabolomics approach. This system provides measurements of more than 180 endogenous metabolites from various classes, including acylcarnitines, amino acids, biogenic amines, glycerophospholipids, and sphingolipids. The AbsoluteIDQ ${ }^{\circledR}$ p180 kit has been validated according to the European Medicine Agency Guidelines on bioanalytical method validation. Several previous studies have used the same platform for MDD participants ${ }^{20,52,64}$. We analyzed de-identified samples following the manufacturer's protocol, with metabolomics labs blinded to clinical data. Amino acids and biogenic amines were analyzed by liquid chromatography (LC) coupled to tandem mass spectrometry, while other metabolites were analyzed using flow-injection analysis (FIA) coupled to tandem mass spectrometry. Identification and quantification were performed based on internal standards and multiple reactions monitoring (MRM) detection. After a pre-processing step (peak integration and concentration determination from calibration curves) with Multiquant software (AB Sciex, Darmstadt, Germany), data were uploaded into Biocrates MetIDQ software (included in the kit). Concentrations of metabolites monitored by FIA were directly calculated in MetIDQ. Detailed sample preparation, metabolite identification and quantification are presented in Supplementary Methods.

\section{Quality control of P180 profiles}

The raw metabolomic profiles included 182 metabolite measurements from 578 participant's plasma samples and 64 quality control samples. Each assay plate contained a group of duplicates that were acquired by combining roughly $10 \mu \mathrm{l}$ from the first 76 samples in the study (QC pool duplicates) to allow for appropriate inter-plate abundance scaling based specifically on this cohort of samples ( $n=24$ across all plates). Quality control steps were taken consistent with our approach in prior publications ${ }^{65}$. Metabolites with $>40 \%$ of measurements below the lower limit of detection (LOD) were excluded from the analysis $(n=163$ metabolites remained in the analysis). Imputation of $\angle \mathrm{LOD}$ values was performed using each metabolite's LOD/2 value to increase statistical power and reduce bias in estimates of the means, consistent with our previous publications. To adjust for batch effects, a correction factor for each metabolite in a specific plate was calculated by dividing metabolites' QC global average by QC average within the plate. Metabolite concentrations were $\log 2$ transformed for statistical analysis. Individuals having both a baseline and a week 8 sample were kept for the subsequent statistical analysis $(n=136$ 
participants). This resulted in an analysis data set containing 272 samples $(n=136$ at baseline and $n=136$ at week 8) and 163 metabolites.

\section{Clinical outcomes}

The $\mathrm{HRSD}_{17}$ total score was used as the outcome measure for both continuous depression symptom severity change and for defining categorical outcomes. Consistent with prior definitions ${ }^{66,67}$, participant outcomes at week 8 were categorized as "remitters" $\left(\mathrm{HRSD}_{17} \leq 7\right)$, "response without remission" $(\geq 50 \%$ reduction from baseline $\mathrm{HRSD}_{17}$, but not reaching remission threshold); "partial response" (30-49\% reduction from baseline $\mathrm{HRSD}_{17}$ score); and "treatment failures" ( $<30 \%$ reduction from baseline $\mathrm{HRSD}_{17}$ score).

\section{Statistical analysis}

Differences in demographic variables and depression scores across the response groups were evaluated using ANOVA and the Pearson Chi-squared test (for categorical variables). All association and differential abundance analyses were performed in a metabolite-wise manner. To examine the significance of log2-fold change in metabolite concentrations, linear mixed-effect models (with random intercept) with $\log 2$ metabolite levels as the dependent variable were fitted while correcting for age, sex, baseline $\mathrm{HRSD}_{17}$, and specific drug (citalopram vs. escitalopram). Then we used the "emmeans" R-package to compute the least squared means of the contrasts of interest (week $8 \mathrm{vs}$. baseline) and their corresponding $P$ values. As a sensitivity analysis, we conducted three additional analyses, stratifying the by sex, age (in three age groups), and drug. Adjustments for multiple comparisons were made using the Benjamini-Hochberg procedure to control the false discovery rate. We investigated the global correlation structure of changing metabolites from baseline to week 8 using Spearman's ranked correlation to identify biochemically related metabolites on which SSRI exposure has a similar effect, followed by hierarchical clustering to group similar correlated metabolites.

To detect whether changes in metabolites were associated with clinical outcomes, we conducted continuous and categorical analyses. In the continuous analysis, the associations of changes in $\mathrm{HRSD}_{17}$ score after 8 weeks with changes in metabolite levels were tested using linear regression models corrected for age and sex. In the categorical analysis, profiles by treatment outcomes were compared using linear mixed-effect models (with random intercept), with $\log 2$ metabolite levels as the dependent variable and the interaction of the week 8 outcome (four level categorical variable: remitters; response without remission; partial response; treatment failures) and visit (two level categorical variable: baseline; week 8) as independent variables while controlling for age, sex, baseline
$\mathrm{HRSD}_{17}$ score, and antidepressant (citalopram/escitalopram), as the method used by other investigators to maximize the ability to identify biological characteristics most clearly associated with differential outcomes ${ }^{67,68}$. Then the fitted models were used to conduct contrasts between the "remission" and "treatment failure" groups at baseline and week 8 using the "emmeans" R-package.

\section{Results}

Demographic and clinical features of the 136 participants are summarized in Table 1. At baseline, age and sex were not significantly different across response groups. Metabolites measured in the p180 kit, as well as QC summary statistics, can be found in Supplementary Table 1 and principal component analysis plots of profiled samples are shown in Supplementary Figs. 1-5.

What are the overall changes in the metabolic profile over 8 weeks of SSRI exposure, and which of these metabolic changes are related to each other?

After 8 weeks of SSRI treatment, levels of several metabolites from different classes were changed significantly ( $q$-value $<0.05$; see Fig. $2 \mathrm{~A}$ and Table 2 ). Among the acylcarnitines, we observed an increased level of three short-chain acylcarnitines (C3, C4, and C5) and a decrease in medium and long-chain acylcarnitines (e.g., C8, C10, C12, C14:2, C16, C16:1, C18, C18:1, and C18:2). Within the classes of amino acids and biogenic amines, we observed a statistically significant increase in two amino acids (arginine and proline) and one biogenic amine (methionine sulfoxide), and a significant decrease in two biogenic amines (serotonin and sarcosine). Among the lipid classes, we observed a statistically significant upregulation of several phosphatidylcholines (PCs) (e.g., PC aa- C36:1, $-\mathrm{C} 30: 0,-\mathrm{C} 42: 2$; ether phosphatidylcholines, $\mathrm{PC}$ ae $\mathrm{C} 34: 3,-\mathrm{C} 38: 2,-\mathrm{C} 36: 3)$ and one sphingolipid (sphingomyelin [SM] C24:0). Figure 2B schematically represents the impact of antidepressant treatment on the interconnected pathways involving polyamines, sarcosine, urea cycle, and 1-carbon metabolism.

Additional stratified analyses based on sex, age group, and drug resulted in similar changes in each stratum (Supplementary Fig. 6 and Supplementary Table 2), indicating that these variables did not meaningfully impact the metabolic changes associated with antidepressant treatment.

As depicted in Fig. 3, correlation analysis revealed that several metabolite changes in response to the drug exposure were highly correlated, especially among metabolites belonging to the same class. We observed five distinct clusters: cluster (1) - mainly formed with medium-chain and long-chain acylcarnitines (e.g., C8, C9, C10, C12, C16); cluster (2)-biogenic amines including serotonin, spermine, spermidine, taurine, putrescine, 
glutamate, ornithine, and sarcosine; cluster (3)-shortchain acylcarnitines $(\mathrm{C} 3, \mathrm{C} 4, \mathrm{C} 5)$, branched-chain amino acids (isoleucine and valine), kynurenine, arginine, glycine, tryptophan, and glutamine; cluster (4)-long-chain ether phospholipids; and cluster (5) - a large number of phospholipids and lysophospholipids.

\section{Which metabolite-level changes are related to changes in depressive symptoms $\left(\mathrm{HRSD}_{17}\right)$ over 8 weeks of SSRI treatment?}

We examined the association of change in $\mathrm{HRSD}_{17}$ with $\log 2$-fold change in metabolite levels from baseline to week 8 , adjusting for the covariates of age and sex. The associations with uncorrected $P$ value $<0.05$ included: one acylcarnitine (C5-M-DC), four amines (histidine, proline, kynurenine, and trans-5 hydroxyproline), seven PC aas and seven PC aes. Change in all of these metabolites was inversely associated with a change in $\mathrm{HRSD}_{17}$ (Supplementary Table 3).

\section{At baseline and after 8 weeks of treatment, which metabolomic profiles differentiate between remitters vs. treatment failures?}

We compared the remitter $(n=64)$ and treatment failure $(n=12)$ groups at baseline and after 8 weeks of SSRI treatment. Eleven metabolites were significantly different (unadjusted $P$ value $<0.05$ ) between the two groups at either baseline or week 8 (Fig. 4 and Supplementary Table 4). Levels of two short-chain acylcarnitines (C3 and $\mathrm{C} 5$ ) and two biogenic amines (alpha-aminoadipic acid and sarcosine) were higher at baseline in the remitters and remained higher at the end of treatment. Serotonin and C3 were significantly different at baseline, but not at week 8. SSRI treatment resulted in differential regulation of four phosphatidylcholines (PC aa C34:2, PC aa C36:2, PC aa C36:4, and PC aa C43:1) and two lysoPCs (lysoPC a C18:2 and lysoPC a C20:4) between the two groups at week 8 .

\section{Discussion}

This study examined the metabolic consequences of 8 weeks of treatment with citalopram or escitalopram in patients with MDD, focusing on acylcarnitines, amino acids, biogenic amines, and lipids. We found significant increases in short-chain acylcarnitines and decreases in the levels of medium-chain and long-chain acylcarnitines. The levels of several amino acids-such as arginine, proline, tyrosine, citrulline, and phenylalanine-were either significantly increased ( $q$-value $<0.05)$ or trended toward an increase (unadjusted $P$ value $<0.05$ ). The biogenic amines serotonin and sarcosine were significantly decreased, while methionine sulfoxide levels increased significantly. Among the lipids, several phosphatidylcholines and ether-phosphatidylcholines were increased 


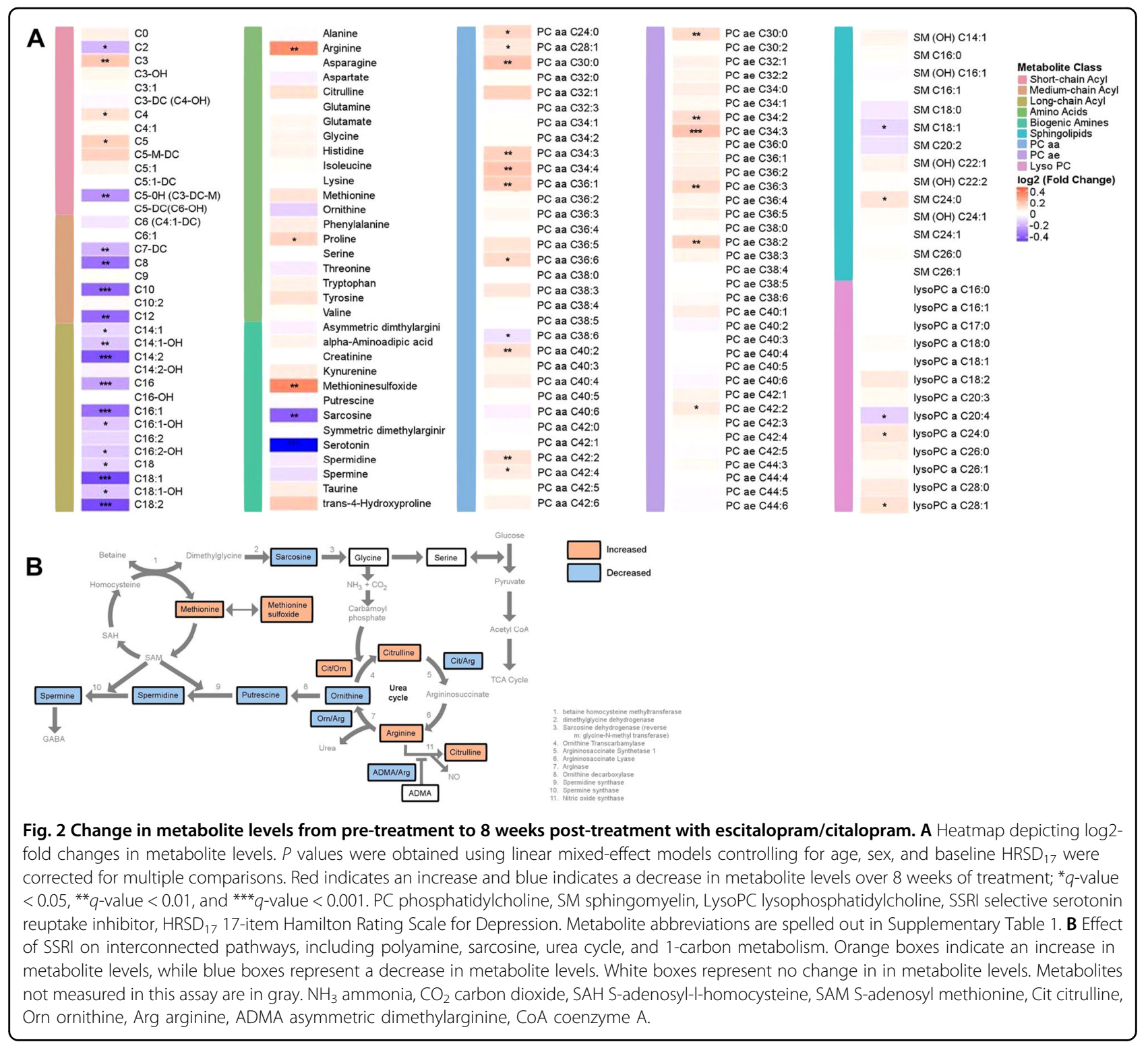

post-treatment which were among the only glycerophospholipids that were assayed in this study. Moreover, changes in the levels of several lipids and amines were correlated with improvements in depression severity $\left(\mathrm{HRSD}_{17}\right)$ after 8 weeks of SSRI treatment. Unique metabolic patterns that differentiate between remitters and patients who failed to respond to the treatment were also noted at baseline and post-treatment. Especially notable were the ether phospholipids that mostly showed an upward trajectory in the remitters while the treatment failure group showed mostly downward trajectories (Supplementary Fig. 7). Notably, the metabolic changes among the patients treated with citalopram versus those treated with escitalopram were very consistent, indicating these drugs have similar metabolomic effects
(Supplementary Fig. 6). The implications of the findings for each group of metabolites is discussed below.

\section{Acylcarnitines}

Acylcarnitines have been implicated in mitochondrial dysfunction and regulation of energy homeostasis in multiple disease models ${ }^{37}$. They play an important role in brain energy homeostasis and cell signaling cascades ${ }^{37}$. Patients with known mitochondrial disorders have frequently reported depressive symptoms ${ }^{25-27}$. Additionally, altered mitochondrial function has been found in patients with a lifetime diagnosis of $\mathrm{MDD}^{23,24,69}$.

Our analysis found that after 8 weeks of SSRI treatment, the short-chain acylcarnitines (specifically propionyl carnitine (C3), butyryl/isobutyryl carnitine (C4), and 


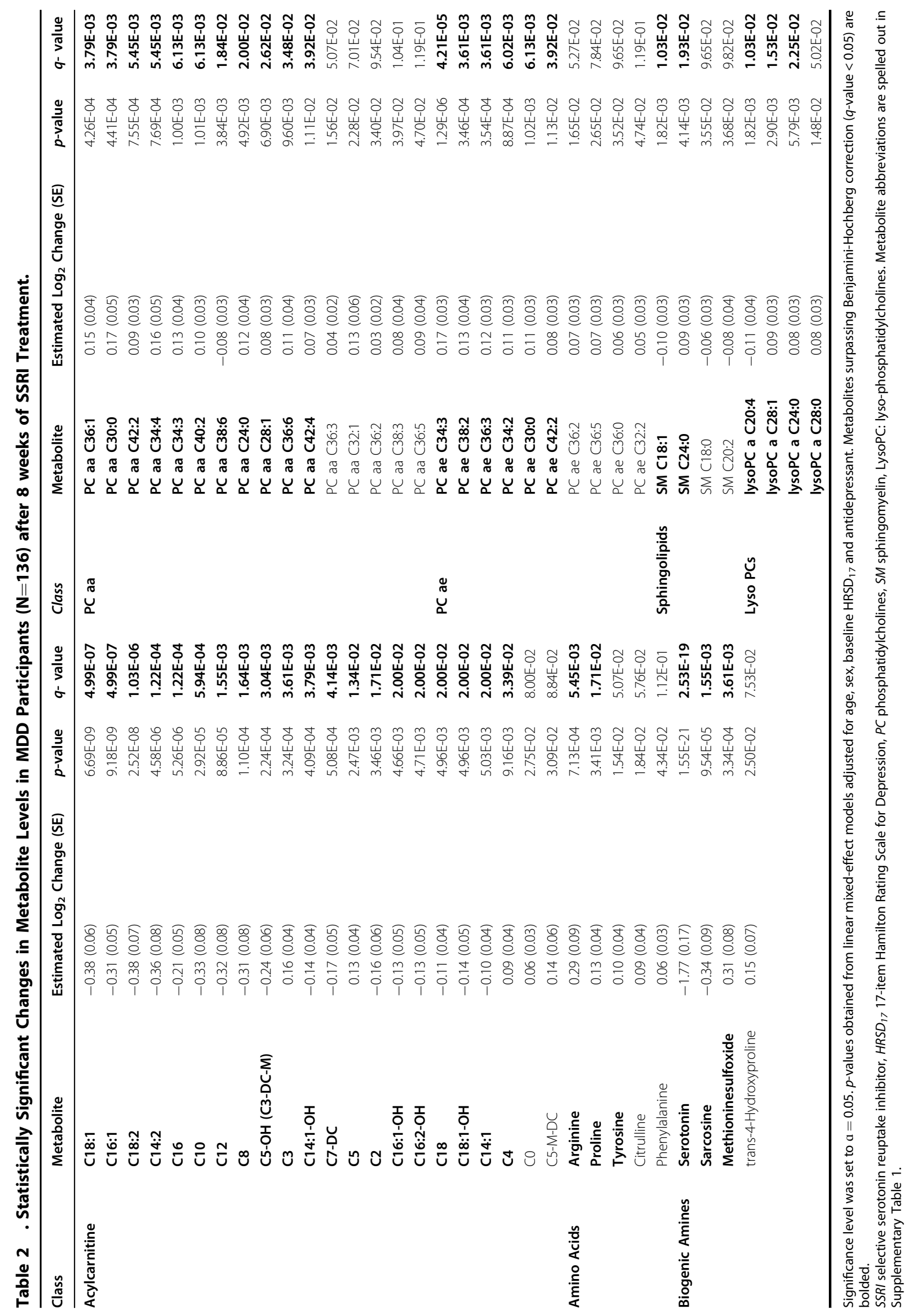




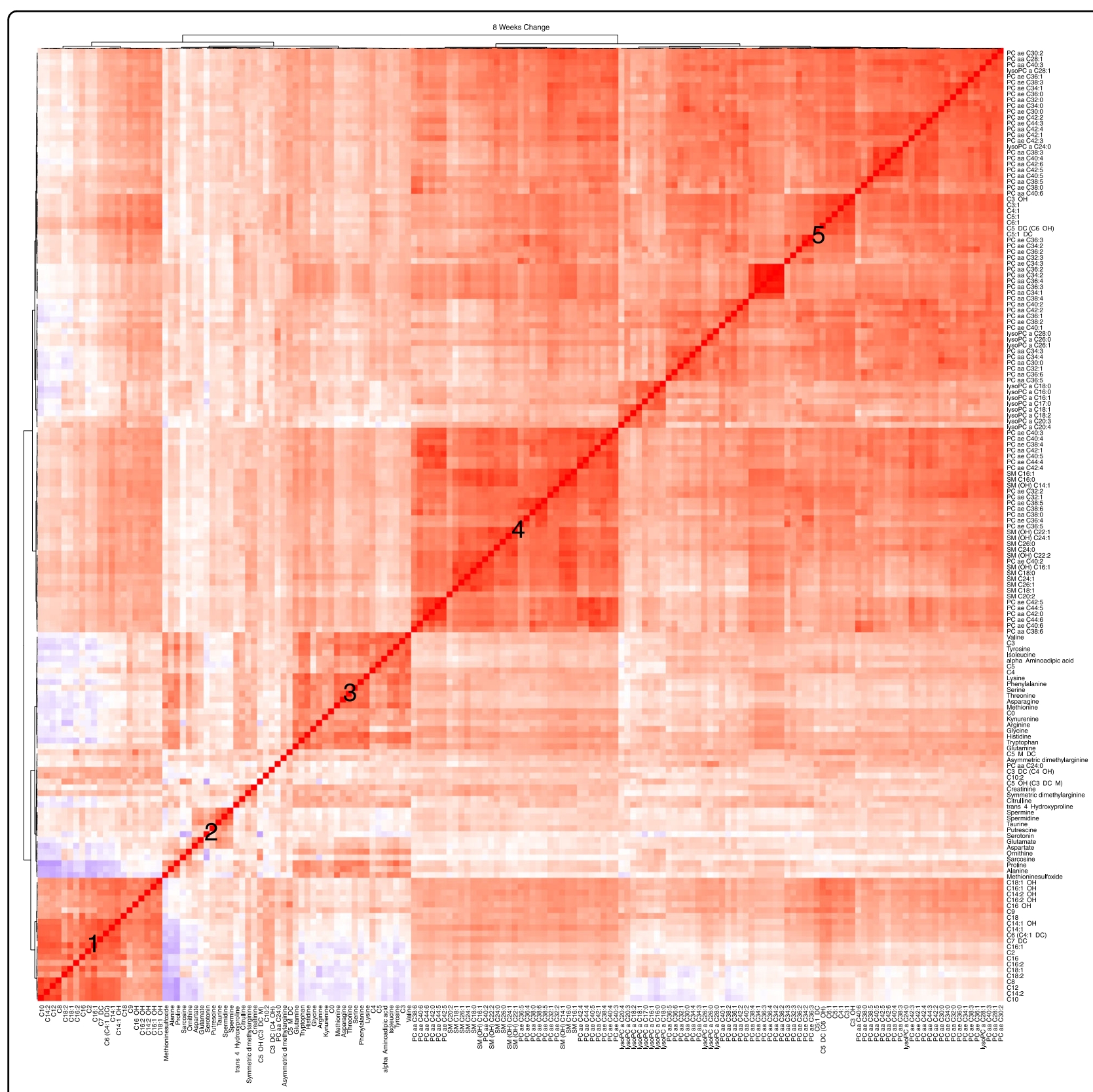

Fig. 3 Hierarchical clustering of Spearman's rank correlation of change in metabolite levels. Red represents positive correlations and blue represents negative correlations. Cluster \#1: enriched for medium and long-chain acylcarnitines (e.g., C8, C9, C10, C12, C16), Cluster \#2: biogenic amines including serotonin, spermine, spermidine, taurine, putrescine, glutamate, ornithine, and sarcosine. Cluster \#3: short-chain-acylcarnitines (C3, C4, C5), branched-chain amino acids (isoleucine and valine), kynurenine, arginine, glycine, tryptophan, and glutamine. Cluster \#4: long-chain phosphodelcholine of PC ae class and Cluster \#5: a large number of phospholipids and lysophospholipids. PC phosphatidylcholine, LysoPC lysophosphatidylcholine, SM sphingomyelin. Metabolite abbreviations are spelled out in Supplementary Table 1.

isovaleryl/methylbutyryl carnitine (C5)) were significantly increased while acetylcarnitine (C2) levels were decreased. Several studies have previously reported perturbations in C2 after antidepressant treatment in depressed patients $^{20,32,52}$. We also observed decreases in mediumchain and long-chain acylcarnitines after 8 weeks of antidepressant therapy and suggest that the drug may act to restore the mitochondrial $\beta$-oxidation process with greater utilization of the medium- and long-chain acylcarnitines. Supporting this interpretation, in a rat model of depression, incomplete $\beta$-oxidation of fatty acids was associated with elevated medium-chain and long-chain 


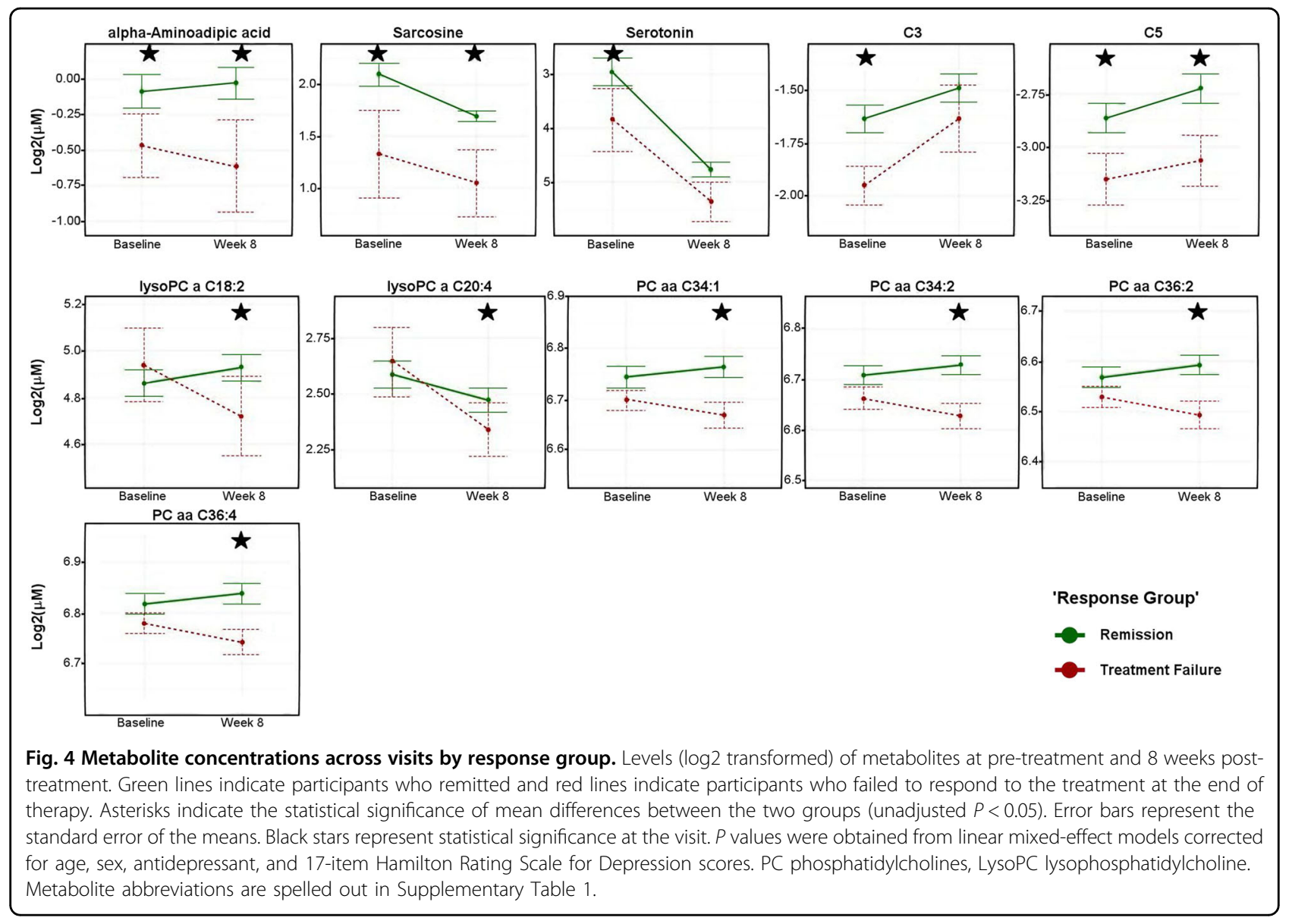

acylcarnitines ${ }^{44}$. Remarkably, in our study, the plasma ratio of long-chain acylcarnitines to free carnitine $(\mathrm{C} 16: 0+\mathrm{C} 18: 0 / \mathrm{C0})$ (which is a marker for the activity of carnitine palmitoyltransferase 1 , the rate-limiting step in the uptake of fatty acids into mitochondria $\left.{ }^{70}\right)$ was significantly reduced at week 8 across the 136 subjects $(P$ value $<3.4 \mathrm{E}-06$ ). In two recent studies that involved schizophrenia patients, similar patterns of short-chain, medium-chain, and long-chain acylcarnitine changes were observed $^{34,35}$, and antipsychotic treatment significantly reduced levels of $\mathrm{C} 2$, increased the short-chain acylcarnitines, and decreased the levels of medium-chain and long-chain acylcarnitines ${ }^{35}$. Taken together, these findings suggest a similar pattern of mitochondrial dysfunction and a drug-induced functional restoration thereof across these two neuropsychiatric disorders.

We also found that the changes in short-chain acylcarnitines (C3, C4, C5) over 8 weeks of SSRI treatment were correlated with changes in branched-chain amino acids (BCAAs; isoleucine and valine). $\mathrm{C} 3$ and $\mathrm{C} 5$ acylcarnitines are products of the degradation of $\mathrm{BCAAs}^{71}$. We and others ${ }^{21,72}$ have previously shown that perturbations in plasma BCAA levels are significantly associated with MDD. BCAAs have well-established anabolic effects on protein metabolism that involve activation of the mTOR pathway ${ }^{21}$ and decreases in BCAAs may result in the dysregulation of the mTOR pathway, leading to depressive symptomology and lower energy metabolism ${ }^{72}$. In our study, we also observed that BCAA levels increased with the antidepressant exposure, though not significantly, and that remitters had higher baseline BCAA levels that further increased post-treatment compared to the treatment failure group.

\section{Amino acids and biogenic amines}

After 8 weeks of SSRI treatment, we noted significant perturbations in the urea cycle and nitric oxide cycle metabolites. Besides clearance of waste nitrogen, the distinct biochemical goals of these cycles involve the production of the intermediates ornithine, citrulline, and arginine for the urea cycle; and polyamine production and the production of nitric oxide for the nitric oxide pathway $^{73}$ (Fig. 2B). In our study, after 8 weeks of drug exposure, plasma arginine levels increased significantly, while ornithine levels showed a trend to be lower compared to baseline. The ratios of citrulline/arginine $(P$ 
value $<0.013)$ and asymmetric dimethylarginine/arginine (ADMA/Arg, $P$ value $<8.62 \mathrm{E}-05$ ) were significantly lower compared to baseline, both of which may indicate potential increases in activity of Nitric Oxide Synthase and increased production of nitric oxide (NO) posttreatment. NO is a known modulator of the major neurotransmitters-norepinephrine, serotonin, dopamine, and glutamate-involved in the neurobiology of $\mathrm{MDD}^{74}$. Rotroff and colleagues have found a negative association between the levels of ornithine and citrulline with changes in the Montgomery-Åsberg depression rating scale after treatment with ketamine ${ }^{20}$. In line with our findings, several studies have reported lower levels of citrulline in MDD patients compared to healthy controls ${ }^{51,52}$, while another reported lower levels of arginine in MDD patients compared to healthy controls ${ }^{53}$. Hence, analyses of these pathways require comprehensive measurement of metabolites rather than an isolated focus on a limited subset.

Similar to our previous findings examining the effects on treatment on neurotransmitters ${ }^{75}$, the biogenic amine serotonin decreased significantly with the drug exposure. A recent study from our group found that after 4 weeks of SSRI exposure, the decrease in sarcosine levels was correlated with the decrease in serotonin levels ${ }^{21}$. Sarcosine is an endogenous amino acid involved in one-carbon metabolism and has recently emerged as a promising therapy for schizophrenia, acting as an N-methyl-D-aspartate receptor agonist $^{76}$. Sarcosine and serotonin were also highly correlated to each other in our study. Additionally, at baseline, their levels were significantly higher in the remitters compared to the treatment failure group and remained higher even at the end of treatment. This suggests that further studies are warranted to determine whether their higher baseline levels were critical to the drug response.

Among the other members of this class, reductions in histidine and kynurenine levels from baseline to week 8 were inversely associated with improvements in depressive symptoms scores HRSD $_{17}$, Supplementary Table 3). Both kynurenine and histidine have been previously implicated in the pathophysiology of depression ${ }^{45,77}$. We also found significant increases in methionine sulfoxide (MetSO; Fig. 2A) and the ratio of methione sulfoxide/ methionine (MetSO/Met; $P$ value $<0.0008$ ) from baseline to week 8 . MetSO is a primary oxidation product of methione, and is a possible biomarker for oxidative stress $^{46}$ which has previously been associated with MDD. Methione residues on proteins can act as sacrificial antioxidants, and the reversible conversion of methione to MetSO may act as a reversible redox switch to regulate the function of proteins ${ }^{78-80}$.

\section{Lipids}

Among the lipids studied, the perturbations amongst the phosphatidylcholines ( $\mathrm{PCs}$ ) containing either the diacyl or the alkyl-acyl moieties were strong, and many of them showed a significant correlation between the reduction in their concentration and improved depression scores (Fig. 2A and Supplementary Table 3). Furthermore, comparisons of remitters versus treatment failure participants revealed several interesting trends. First, the lysophosphatidylcholines containing a single fatty acyl chain (lysoPCs -C16:0, -C17:0, -C18:0, -C18:1, C18:2, $\mathrm{C} 20: 3$, and C20:4) mostly showed downward trajectories from baseline to week 8 among the treatment failure group but not among the remitters. Second, several diacyl PCs that showed inverse correlations to changes in depression scores (Fig. 4 and Supplementary Table 3) (e.g., PC aa -C34:1, 34:2, 36:2, 36:3, 36:4, 38:4) showed very similar trajectories in that the remitters all had higher baseline levels that increased further at week 8 , compared to the treatment failure group which showed very similar downward trajectories (Fig. 4). Lastly, the ether-phosphatidylcholines as a group showed a distinct pattern of perturbation: the treatment failure group mostly had significantly higher baseline levels compared to the remitters, and these metabolites then followed downward trajectories over the treatment period whereas the remitters either showed an increase or stayed unchanged (Supplementary Fig. 7). Overall, the lipids displayed remarkable differences between the remitters and treatment failure group regarding how the two groups responded to the drug. While it is difficult to come up with plausible explanations for such observed differences, there seems to be an important role for these lipids in contributing to drug response.

Lipids are involved in crucial brain functions, including cell membrane structure, membrane transmitters, energy metabolism, and neuroendocrine function ${ }^{59}$. Blood lipid profile changes have been implicated in the pathophysiology of depression, schizophrenia, and Alzheimer's disease $^{54-58}$. Associations between altered in lipid profiles and the presence of depression and anxiety have been previously reported in several studies ${ }^{81-85}$.

Of special interest is the distinct pattern, we observed among the ether phospholipids. The distinctive chemical feature of the ether lipids is the ether bond at the $s n-1$ position of the glycerol backbone where fatty alcohol is attached as opposed to the more common diacyl moiety containing phospholipids. The emerging role of these lipids in neurological diseases like Alzheimer's disease and autism is currently garnering much interest ${ }^{86}$. They act as antioxidants and are essential in shaping membrane integrity and properties, thereby potentially impacting numerous biological processes and functions. Recently, ether-lipid-deficient knock-out mice demonstrated behavioral alterations and reduced brain levels of various neurotransmitters, which the investigators attributed to altered synaptic vesicle function ${ }^{87,88}$. Recently, Knowles 
et al. demonstrated a shared genetic association between MDD and ether-phosphatidylcholine species that contain arachidonic acid, the latter being a precursor to the proinflammatory prostaglandins ${ }^{89}$. The initial stages of ether-lipid synthesis occur in the peroxisomes, including the rate-limiting enzymatic processes. It is also possible that peroxisomal disorders and subsequent metabolic resilience result in the distinct patterns observed in the trajectories of ether-phospholipids in remitters vs. those in the treatment failure group.

We also noted perturbation of phospholipid profiles. Additionally, remitters show unique changes in their lipid profiles compared to participants with treatment failure. Although only countable PC species are significantly changed in the remitter group, essentially all PC species containing saturated or monounsaturated fatty acyl chains (i.e., PC species containing $\leq 3$ total double bonds) tended to increase, whereas PC species containing $\geq 4$ total double bonds were reduced or tended toward reduction. These changes were not observed in the treatment failure group. These types of changes likely resulted from the remodeling of PC species, probably in the liver, through a combined action of phospholipases (e.g., phospholipase A2) and acyltransferase activities. Although the activity of lecithin cholesteryl ester transferase could also contribute to this change, this activity yields less selective remodeling of fatty acyl chains.

The changes in the acylcarntines and PC species in the remitter group could be inter-related. For example, the accumulation of palmitate, oleate, and their relevant fatty acyls due to a reduced CPT1 activity could lead to maladaptive changes of the PC pattern due to reduced precursor availability for acyltransferase activity, as uncovered in this study. Alternatively, remodeling of PC species due to the action of SSRI treatment may produce changes in membrane structure and function, thus affecting the activities of membrane proteins such as CPT1. However, the cause-and-effect relationship remains unknown. Further studies to clarify this relationship-such as measuring the inhibitory effect of SSRI on CPT1 activities and determining the accumulation of specific acylCoAs-are clearly needed.

The above information shows that assessing metabolic profiles may enable the mapping of global biochemical changes in MDD, provide a means to characterize the remitted and depressed states and provide a way of characterizing the effects of antidepressants on metabolic pathways. This approach may ultimately inform therapeutic choices, thus reducing trial-and-error prescribing and contributing to personalizing therapeutic treatment for patients with MDD.

This study has several limitations. First, some of the investigated acylcarnitines-especially dicarboxylic and hydroxylated species (C3-DC, C5-DC, C5-MD-C, C16:
$\mathrm{OH}, \mathrm{C} 18: \mathrm{OH}$ - are recognizably high only in patients with rare inborn errors of metabolism, and display undetectable concentrations in most individuals. In this report, the flow-injection MS/MS method used to measure acylcarnitines lacks the specificity to confirm exact structures for low-level or isobaric species such as "C5 acylcarnitine" (which is the sum of isovaleryl and 2methylbutyryl carnitine). Nevertheless, the measures reported here showed exceptional technical reproducibility. Also, for many of the low-abundance (i.e., $<0.1 \mu \mathrm{M}$ ) acylcarnitines, our average observed values were below the pathological clinical reference threshold described by Mayo Clinic Laboratories ${ }^{90}$. The low-abundance acylcarnitines reported here may benefit from utilization of an assay with greater molecular specificity to confirm the exact molecular speciation, such as a research-grade LCMS/MS assay reported previously ${ }^{91}$. We had limited ability to characterize the fatty acid moieties bound to the phospholipids, which warrants a detailed future study on the lipids, especially given the fact that the polyunsaturated fatty acids have been shown to impact multiple neuropsychiatric diseases including major depression. Another limitation is the relatively small sample size of the study and unbalanced sample sizes in the remission and treatment-failure groups. It will be necessary to replicate and validate our findings in larger independent studies. Further, the lack of a control or placebo group limits our ability to control for spontaneous changes in the acylcarnitine profiles that occur regardless of treatment or the passage of time. In addition, plasma samples were in a non-fasting state which can lead to variability in metabolite levels. Diet, medication, and other factors may also contribute to changes in metabolite levels over time.

To summarize, our data suggest that SSRI treatment results in changes in the acylcarnitine, lipid, and amino acid profiles. The altered metabolic profiles suggest that mitochondrial energetics are implicated in recovery from a depressed state. The changes in acylcarnitine profiles were similar to a handful of studies conducted in other psychiatric illnesses, such as first-episode psychosis, which suggests a substantial overlap of implicated pathways and mechanisms across mental illnesses. The data show that after treatment, long-chain acylcarnitines are reduced but short-chain acylcarnitines are increased. The reduced medium-chain and long-chain fatty acylcarnitine levels could have resulted from enhanced mitochondrial fatty acid oxidation after treatment. However, further studies are needed to definitively clarify these possibilities. Whatever the case, it appears that reduced efficiency of ATP production is likely associated with MDD. This finding could be further explored to develop new therapeutics targeting other approaches to restore balance in energy production. More importantly, a reduced CPT1 
activity could lead to the accumulation of palmitate, oleate, and their relevant fatty acylCoA species since CPT1 is relatively selective for transporting these two fatty acyls to mitochondria. These and similar data demonstrate the power of metabolomics for understanding disease mechanisms, the molecular basis of disease heterogeneity, and variation of response to treatment and warrant validation in larger studies.

\begin{abstract}
Acknowledgements
The authors are grateful for the support of $\mathrm{NIH}$, to Lisa Howerton for her administrative support, to David S. Millington, PhD for insightful discussions and suggestions, and to the study participants and their families of the Mayo Pharmacogenomics Research Network-Antidepressant Pharmacogenomics Medication Study (PGRN-AMPS). The research and the authors are supported by funding from the $\mathrm{NIH}$. This work was funded by grant support to Rima Kaddurah-Daouk through NIH grants R01MH108348, R01AG046171 \& U01AG061359, RF1AG051550. R.M.W. was supported by NIH grants RO1 GM28157, U19 GM61388, U54 GM114838, and NSF1624615. S.B. was supported by the $\mathrm{NIH}$ grant R01MH108348.
\end{abstract}

\section{Author details}

'Department of Psychiatry and Behavioral Sciences, Duke University School of Medicine, Durham, Durham, NC, USA. ${ }^{2}$ Department of Neurology, Mayo Clinic, Rochester, MN, USA. ${ }^{3}$ Department of Biological Sciences and Arkansas Biosciences Institute, Arkansas State University, Jonesboro, AR, USA. ${ }^{4}$ University of Texas Health Science Center at San Antonio, San Antonio, TX, USA. ${ }^{5}$ Rosa \& Co LLC, San Carlos, CA, USA. Institute of Bioinformatics and Systems Biology, Helmholtz Zentrum München, German Research Center for Environmental Health, Neuherberg, Germany. 'Department of Psychiatry and Psychology, Mayo Clinic, Rochester, MN, USA. ${ }^{8}$ Proteomics and Metabolomics Shared Resource, Center for Genomic and Computational Biology, Duke University, Durham, NC 27710, USA. ${ }^{9}$ Department of Psychiatry and Behavioral Sciences, Emory University School of Medicine, Atlanta, GA, USA. ${ }^{10}$ Department of Psychiatry, Rush Medical College, Chicago, IL, USA. ${ }^{1}$ Professor Emeritus, Department of Pediatrics, Duke University School of Medicine, Durham, NC, USA. ${ }^{12}$ Department of Psychiatry, Texas Tech University, Health Sciences Center, Permian Basin, TX, USA. ${ }^{13}$ Department of Molecular Pharmacology \& Experimental Therapeutics, Mayo Clinic, Rochester, MN, USA. ${ }^{14}$ Department of Medicine, Duke University, Durham, NC, USA. ${ }^{15}$ Duke Institute of Brain Sciences, Duke University, Durham, NC, USA

\section{Conflict of interest}

R.M.W. is a cofounder and stockholder in OneOme, LLC, a pharmacogenomic clinical decision-support company. A.J.R. has received consulting fees from Akili, Brain Resource Inc., Compass Inc., Curbstone Consultant LLC., Emmes Corp., Johnson and Johnson (Janssen), Liva-Nova, Mind Linc, Sunovion, and Taj Medical; speaking fees from Liva-Nova; and royalties from Guilford Press and the University of Texas Southwestern Medical Center, Dallas, TX (for the Inventory of Depressive Symptoms and its derivatives). He has also named the coinventor on two patents: US Patent No. 7,795,033: Methods to Predict the Outcome of Treatment with Antidepressant Medication and US Patent No. 7,906,283: Methods to Identify Patients at Risk of Developing Adverse Events during Treatment with Antidepressant Medication. M.A.F. has received grant support from AssureRx Health Inc, Myriad, Pfizer Inc, NIMH (R01 MH079261), the National Institute on Alcohol Abuse and Alcoholism (P20AA017830) in the National Institutes of Health at the US Department of Health and Human Services, and the Mayo Foundation. He has been a consultant (for Mayo) to Janssen Global Services, LLC; Mitsubishi Tanabe Pharma Corp; Myriad Genetics, Inc; Sunovion Pharmaceuticals, Inc; and Teva Pharmaceutical Industries Ltd. He has received a continuing medical education, travel, and presentation support from the American Physician Institute and CME Outfitters. R.K.-D. is an inventor on key patents in the field of metabolomics. M.A. was supported by the National Institute on Aging [R01AG057452, RF1AG051550, and R01AG046171], National Institute of Mental Health [R01MH108348], and Qatar National Research Fund [NPRP8-061-3-011]. The funders listed above had no role in the design and conduct of the study; collection, management, analysis, and interpretation of the data; preparation, review, or approval of the paper; and decision to submit the paper for publication.

\section{Publisher's note}

Springer Nature remains neutral with regard to jurisdictional claims in published maps and institutional affiliations.

Supplementary information Supplementary Information accompanies this paper at (https://doi.org/10.1038/s41398-020-01097-6).

Received: 4 February 2020 Revised: 1 October 2020 Accepted: 26 October 2020

Published online: 02 March 2021

\section{References}

1. World Health Organization, W. "Depression: let's talk" says WHO, as depression tops list of causes of ill health. http://www.who.int/mediacentre/news/ releases/2017/world-health-day/en/ (2017).

2. Kennedy, S. H. et al. Canadian network for mood and anxiety treatments (CANMAT) 2016 clinical guidelines for the management of adults with major depressive disorder: section 3. Pharmacological treatments. Can. J. Psychiatry 61, 540-560 (2016).

3. Anderson, I. M. et al. Evidence-based guidelines for treating depressive disorders with antidepressants: a revision of the 2000 British association for psychopharmacology guidelines. J. Psychopharmacol. 22, 343-396 (2008).

4. Rush, A. J. et al. Bupropion-SR, sertraline, or venlafaxine-XR after failure of SSRls for depression. N. Engl. J. Med. 354, 1231-1242 (2006).

5. Rush, A. J. et al. Selecting among second-step antidepressant medication monotherapies: predictive value of clinical, demographic, or first-step treatment features. Arch. Gen. Psychiatry 65, 870-880 (2008).

6. Rush, A. J. et al. Acute and longer-term outcomes in depressed outpatients requiring one or several treatment steps: a STAR*D report. Am. J. Psychiatry 163, 1905-1917 (2006).

7. Kaddurah-Daouk, R. et al. Cerebrospinal fluid metabolome in mood disordersremission state has a unique metabolic profile. Sci. Rep. 2, 667 (2012).

8. Paige, L. A., Mitchell, M. W., Krishnan, K. R., Kaddurah-Daouk, R. \& Steffens, D. C. A preliminary metabolomic analysis of older adults with and without depression. Int. J. Geriatr. Psychiatry 22, 418-423 (2007).

9. Steffens, D. C. et al. Metabolomic differences in heart failure patients with and without major depression. J. Geriatr. Psychiatry Neurol. 23, 138-146 (2010).

10. Gadad, B. S. et al. Peripheral biomarkers of major depression and antidepressant treatment response: current knowledge and future outlooks. $J$. Affect. Disord. 233, 3-14 (2018).

11. MacDonald, $K$. et al. Biomarkers for major depressive and bipolar disorders using metabolomics: a systematic review. Am. J. Med. Genet. Part B, Neuropsychiatric Genet. 180, 122-137 (2019).

12. Pedrini, M. et al. Advances and challenges in development of precision psychiatry through clinical metabolomics on mood and psychotic disorders. Prog. Neuro-psychopharmacol. Biol. Psychiatry 93, 182-188 (2019).

13. Wenk, M. R. The emerging field of lipidomics. Nat. Rev. Drug Discov. 4, 594-610 (2005).

14. Griffin, J. L. The Cinderella story of metabolic profiling: does metabolomics get to go to the functional genomics ball? Philos. Trans. R. Soc. Lond. Series B, Biol. Sci. 361, 147-161 (2006).

15. Kaddurah-Daouk, R. \& Krishnan, K. R. Metabolomics: a global biochemical approach to the study of central nervous system diseases. Neuropsychopharmacology 34, 173-186 (2009).

16. Kaddurah-Daouk, R. \& Weinshilboum, R. Metabolomic signatures for drug response phenotypes: pharmacometabolomics enables precision medicine. Clin. Pharmacol. Ther. 98, 71-75 (2015).

17. Kaddurah-Daouk, R. \& Weinshilboum, R. M. Pharmacometabolomics: implications for clinical pharmacology and systems pharmacology. Clin. Pharmacol. Ther. 95, 154-167 (2014).

18. Beger, R. D. et al. Metabolomics enables precision medicine: "a white paper, community perspective". Metabolomics 12, 149-149 (2016).

19. Zhu, H. et al. Pharmacometabolomics of response to sertraline and to placebo in major depressive disorder-possible role for methoxyindole pathway. PLOS ONE 8, e68283 (2013). 
20. Rotroff, D. M. et al. Metabolomic signatures of drug response phenotypes for ketamine and esketamine in subjects with refractory major depressive disorder: new mechanistic insights for rapid acting antidepressants. Transl. Psychiatry 6, e894 (2016).

21. Kaddurah-Daouk, R. et al. Pharmacometabolomic mapping of early biochemical changes induced by sertraline and placebo. Transl. Psychiatry 3, e223 (2013).

22. Kaddurah-Daouk, R. et al. Pretreatment metabotype as a predictor of response to sertraline or placebo in depressed outpatients: a proof of concept. Transl. Psychiatry 1, https://doi.org/10.1038/tp.2011.22 (2011).

23. Gardner, A. et al. Alterations of mitochondrial function and correlations with personality traits in selected major depressive disorder patients. J. Affect. Disord. 76, 55-68 (2003).

24. Beasley, C. L. et al. Proteomic analysis of the anterior cingulate cortex in the major psychiatric disorders: evidence for disease-associated changes. Proteomics 6, 3414-3425 (2006).

25. Kato, T. The other, forgotten genome: mitochondrial DNA and mental disorders. Mol. Psychiatry 6, 625-633 (2001).

26. Fattal, O., Link, J., Quinn, K., Cohen, B. H. \& Franco, K. Psychiatric comorbidity in 36 adults with mitochondrial cytopathies. CNS Spectrums 12, 429-438 (2007).

27. Manji, H. et al. Impaired mitochondrial function in psychiatric disorders. Nat. Rev. Neurosci. 13, 293-307 (2012)

28. Wang, W. et al. Rapid-acting antidepressant-like effects of acetyl-I-carnitine mediated by PI3K/AKT/BDNFNGF signaling pathway in mice. Neuroscience 285, 281-291 (2015).

29. Wang, S. M. et al. A review of current evidence for acetyl---carnitine in the treatment of depression. J. Psychiatric Res. 53, 30-37 (2014).

30. Nasca, C. et al. L-acetylcarnitine causes rapid antidepressant effects through the epigenetic induction of mGlu2 receptors. Proc. Natl Acad. Sci. USA 110, 4804-4809 (2013).

31. Nasca, D., G. Zurria, G. \& Aguglia, E. Action of Acetyl-L-carnitine in Association with Mianserine on Depressed Old People. New Trends Clin Neuropharmacol. 3, 225-230 (1989)

32. Nasca, C. et al. Acetyl-I-carnitine deficiency in patients with major depressive disorder. Proc. Natl Acad. Sci. USA 115, 8627-8632 (2018).

33. Frye, R. E., Melnyk, S. \& Macfabe, D. F. Unique acyl-carnitine profiles are potential biomarkers for acquired mitochondrial disease in autism spectrum disorder. Transl. Psychiatry 3, e220 (2013).

34. Kriisa, K. et al. Profiling of acylcarnitines in first episode psychosis before and after antipsychotic treatment. J. Proteome Res. 16, 3558-3566 (2017).

35. Cao, B. et al. Characterizing acyl-carnitine biosignatures for schizophrenia: a longitudinal pre- and post-treatment study. Transl. Psychiatry 9, 19 (2019).

36. Fritz, I. B. \& Mcewen, B. Effects of carnitine on fatty-acid oxidation by muscle. Science 129, 334-335 (1959).

37. Jones, L. L., McDonald, D. A. \& Borum, P. R. Acylcarnitines: role in brain. Prog. Lipid Res. 49, 61-75 (2010).

38. Bieber, L. L. Carnitine. Annu. Rev. Biochem. 57, 261-283 (1988).

39. Rinaldo, P., Cowan, T. M. \& Matern, D. Acylcarnitine profile analysis. Genet. Med. 10, 151-156 (2008).

40. Kler, R. S. et al. Quantitation of acyl-CoA and acylcarnitine esters accumulated during abnormal mitochondrial fatty acid oxidation. J. Biol. Chem. 266, 22932-22938 (1991)

41. Vreken, P. et al. Quantitative plasma acylcarnitine analysis using electrospray tandem mass spectrometry for the diagnosis of organic acidaemias and fatty acid oxidation defects. J. Inherit. Metab. Dis. 22, 302-306 (1999).

42. Wanders, R. J. et al. Disorders of mitochondrial fatty acyl-CoA beta-oxidation. J. Inherit. Metab. Dis. 22, 442-487 (1999).

43. Cavedon, C. T. et al. Age-related variations in acylcarnitine and free carnitine concentrations measured by tandem mass spectrometry. Clin. Chem. 51, 745-752 (2005).

44. Chen, S. et al. Effect of Allium macrostemon on a rat model of depression studied by using plasma lipid and acylcarnitine profiles from liquid chromatography/mass spectrometry. J. Pharm. Biomed. Anal. 89, 122-129 (2014).

45. Adachi, Y. et al. Association between plasma alpha-aminobutyric acid and depressive symptoms in older community-dwelling adults in Japan. Geriatr. Gerontol. Int. https://doi.org/10.1111/ggi.13585 (2018).

46. Mashima, R., Nakanishi-Ueda, T. \& Yamamoto, Y. Simultaneous determination of methionine sulfoxide and methionine in blood plasma using gas chromatography-mass spectrometry. Anal. Biochem. 313, 28-33 (2003).
47. Smaga, I. et al. Oxidative stress as an etiological factor and a potential treatment target of psychiatric disorders. Part 2. Depression, anxiety, schizophrenia and autism. Pharmacol. Rep. 67, 569-580 (2015).

48. Maurya, P. K. et al. The role of oxidative and nitrosative stress in accelerated aging and major depressive disorder. Prog. Neuro-psychopharmacol. Biol. Psychiatry 65, 134-144 (2016).

49. Morris, S. M. Jr Arginine: beyond protein. Am. J. Clin. Nutr. 83, 508s-512s (2006).

50. Wegener, G. \& Volke, V. Nitric oxide synthase inhibitors as antidepressants. Pharmaceuticals 3, 273-299 (2010).

51. Hess, S. et al. Decreased serum L-arginine and L-citrulline levels in major depression. Psychopharmacology 234, 3241-3247 (2017).

52. Moaddel, R. et al. Plasma metabolomic profiling of a ketamine and placebo crossover trial of major depressive disorder and healthy control subjects. Psychopharmacology 235, 3017-3030 (2018)

53. Ali-Sisto, T. et al. Global arginine bioavailability ratio is decreased in patients with major depressive disorder. J. Affect. Disord. 229, 145-151 (2018).

54. Demirkan, A. et al. Plasma phosphatidylcholine and sphingomyelin concentrations are associated with depression and anxiety symptoms in a Dutch family-based lipidomics study. J. Psychiatric Res. 47, 357-362 (2013).

55. Kakefuda, K. et al. Diacylglycerol kinase beta knockout mice exhibit lithiumsensitive behavioral abnormalities. PLOS ONE 5, e13447 (2010).

56. Liu, X. et al. Discovery and validation of plasma biomarkers for major depressive disorder classification based on liquid chromatography-mass spectrometry. J. Proteome Res 14, 2322-2330 (2015).

57. McEvoy, J. et al. Lipidomics reveals early metabolic changes in subjects with schizophrenia: effects of atypical antipsychotics. PLOS ONE 8, e68717 (2013).

58. Kasukawa, T. et al. Human blood metabolite timetable indicates internal body time. Proc. Natl Acad. Sci. USA 109, 15036-15041 (2012).

59. Fonteh, A. N. et al. Identification of disease markers in human cerebrospinal fluid using lipidomic and proteomic methods. Dis. Markers 22, 39-64 (2006)

60. Harrison, P. J. The neuropathology of primary mood disorder. Brain: J. Neurol. 125, 1428-1449 (2002).

61. Muller, C. P. et al. Brain membrane lipids in major depression and anxiety disorders. Biochimica et Biophysica Acta 1851, 1052-1065 (2015).

62. Mrazek, D. A. et al. Treatment outcomes of depression: the pharmacogenomic research network antidepressant medication pharmacogenomic study. J. Clin. Psychopharmacol. 34, 313-317 (2014).

63. Hamilton, M. Development of a rating scale for primary depressive illness. Br. J. Soc. Clin. Psychology 6, 278-296 (1967).

64. Baranyi, A. et al. Metabolomics approach in the investigation of depression biomarkers in pharmacologically induced immune-related depression. PLOS ONE 13, e0208238-e0208238 (2018).

65. St John-Williams, L. et al. Targeted metabolomics and medication classification data from participants in the ADNI1 cohort. Sci. Data 4, 170140 (2017).

66. Rush, A. J. et al. Report by the ACNP task force on response and remission in major depressive disorder. Neuropsychopharmacology 31, 1841-1853 (2006).

67. Dunlop, B. W. et al. Effects of patient preferences on outcomes in the predictors of remission in depression to individual and combined treatments (PReDICT) study. Am. J. Psychiatry 174, 546-556 (2017).

68. Vadodaria, K. C. et al. Serotonin-induced hyperactivity in SSRI-resistant major depressive disorder patient-derived neurons. Mol. Psychiatry https://doi.org/ 10.1038/s41380-019-0363-y (2019).

69. Suomalainen, A. et al. Multiple deletions of mitochondrial DNA in several tissues of a patient with severe retarded depression and familial progressive external ophthalmoplegia. J. Clin. Investig. 90, 61-66 (1992).

70. Heiner-Fokkema, M. R. et al. Reliable diagnosis of carnitine palmitoyltransferase type IA deficiency by analysis of plasma acylcarnitine profiles. JIMD Rep. 32, 33-39 (2017).

71. Schooneman, M. G., Vaz, F. M., Houten, S. M. \& Soeters, M. R. Acylcarnitines: reflecting or inflicting insulin resistance? Diabetes 62, 1-8 (2013).

72. Baranyi, A. et al. Branched-chain amino acids as new biomarkers of major depression - a novel neurobiology of mood disorder. PLOS ONE 11, e0160542 (2016).

73. Neill, M. A., Aschner, J., Barr, F. \& Summar, M. L. Quantitative RT-PCR comparison of the urea and nitric oxide cycle gene transcripts in adult human tissues. Mol. Genet. Metab. 97, 121-127 (2009).

74. Dhir, A. \& Kulkarni, S. K. Nitric oxide and major depression. Nitric Oxide 24, 125-131 (2011).

75. Bhattacharyya, S. et al. Metabolomic signature of exposure and response to citalopram/escitalopram in depressed outpatients. Transl. Psychiatry 9, 173 (2019). 
76. Zhang, H. X., Lyons-Warren, A. \& Thio, L. L. The glycine transport inhibitor sarcosine is an inhibitory glycine receptor agonist. Neuropharmacology 57, 551-555 (2009).

77. Reus, G. Z. et al. Kynurenine pathway dysfunction in the pathophysiology and treatment of depression: evidences from animal and human studies. J. Psychiatric Res. 68, 316-328 (2015).

78. Levine, R. L., Berlett, B. S., Moskovitz, J., Mosoni, L. \& Stadtman, E. R. Methionine residues may protect proteins from critical oxidative damage. Mech. Ageing Dev. 107, 323-332 (1999).

79. Levine, R. L., Moskovitz, J. \& Stadtman, E. R. Oxidation of methionine in proteins: roles in antioxidant defense and cellular regulation. IUBMB Life $\mathbf{5 0}$, 301-307 (2000).

80. Davies, M. J. The oxidative environment and protein damage. Biochimica et Biophysica Acta 1703, 93-109 (2005).

81. Huang, T. L., Wu, S. C., Chiang, Y. S. \& Chen, J. F. Correlation between serum lipid, lipoprotein concentrations and anxious state, depressive state or major depressive disorder. Psychiatry Res. 118, 147-153 (2003).

82. Papakostas, G. I. et al. Serum cholesterol and serotonergic function in major depressive disorder. Psychiatry Res. 118, 137-145 (2003).

83. Sagud, M., Mihaljevic-Peles, A., Pivac, N., Jakovljevic, M. \& Muck-Seler, D. Lipid levels in female patients with affective disorders. Psychiatry Res. 168, 218-221 (2009).
84. Sahebzamani, F. M. et al. Relationship among low cholesterol levels, depressive symptoms, aggression, hostility, and cynicism. J. Clin. Lipidol. 7, 208-216 (2013).

85. van Reedt Dortland, A. K. et al. Associations between serum lipids and major depressive disorder: results from the Netherlands Study of Depression and Anxiety (NESDA). J. Clin. Psychiatry 71, 729-736 (2010).

86. Dorninger, F., Forss-Petter, S. \& Berger, J. From peroxisomal disorders to common neurodegenerative diseases - the role of ether phospholipids in the nervous system. FEBS Lett. 591, 2761-2788 (2017)

87. Dorninger, F., Gundacker, A., Zeitler, G., Pollak, D. D. \& Berger, J. Ether lipid deficiency in mice produces a complex behavioral phenotype mimicking aspects of human psychiatric disorders. Int. J. Mol. Sci. 20, https://doi.org/ 10.3390/ijms20163929 (2019).

88. Dorninger, F. et al. Disturbed neurotransmitter homeostasis in ether lipid deficiency. Hum. Mol. Genet. 28, 2046-2061 (2019).

89. Knowles, E. E. M. et al. The lipidome in major depressive disorder: shared genetic influence for ether-phosphatidylcholines, a plasma-based phenotype related to inflammation, and disease risk. Eur. Psychiatry 43, 44-50 (2017).

90. Mayo Clinic Laboratories. Acylcarnitines, Quantitative, Plasma. https://www. mayocliniclabs.com/test-catalog/Clinical+and+Interpretive/82413 (2017).

91. Minkler, P. E., Stoll, M. S., Ingalls, S. T., Kerner, J. \& Hoppel, C. L. Quantitative acylcarnitine determination by UHPLC-MS/MS-Going beyond tandem MS acylcarnitine "profiles". Mol. Genet. Metab. 116, 231-241 (2015).

\section{Author details}

\section{The Mood Disorders Precision Medicine Consortium (MDPMC)}

Rima Kaddurah-Daouk ${ }^{16}$, John Rush ${ }^{16}$, Jessica Tenenbaum ${ }^{16}$, Arthur Moseley ${ }^{16}$, Will Thompson ${ }^{16}$, Gregory Louie ${ }^{16}$, Colette Blach ${ }^{16}$, Siamak Mahmoudiandehkhordi ${ }^{16}$, Rebecca Baillie ${ }^{17}$, Xianlin Han ${ }^{18}$, Sudeepa Bhattacharyya ${ }^{19}$, Mark Frye ${ }^{20}$, Richard Weinshilboum ${ }^{20}$, Ahmed Ahmed $^{20}$, Drew Neavin ${ }^{20}$, Duan Liu $^{20}$, Michelle Skime ${ }^{20}$, Piero Rinaldo ${ }^{20}$, Oliver Fiehn ${ }^{21}$, Christopher Brydges ${ }^{21}$, Helen Mayberge ${ }^{22}$, Ki Sueng Choi ${ }^{22}$, Jungho Cha ${ }^{22}$, Gabi Kastenmüller ${ }^{23}$, Matthias Arnold ${ }^{23}$, Elisabeth Binder ${ }^{24}$, Janine Knauer-Arloth ${ }^{24}$, Alejo Nevado-Holgado ${ }^{25}$, Liu Shi ${ }^{25}$, Boadie Dunlop ${ }^{26}$, Ed Craighead ${ }^{26}$, William McDonald ${ }^{26}$, Patricio Riva Posse ${ }^{26}$, Brenda Pennin $x^{27}$, Yuri Milaneschi ${ }^{27}$, Rick Jansen ${ }^{27}$ and Ranga Krishnan ${ }^{28}$

\footnotetext{
${ }^{16}$ Duke University Medical Center, Durham, NC, USA. ${ }^{17}$ Rose \& Co, San Carlos, CA, USA. ${ }^{18}$ UT Health - San Antonio, San Antonio, TX, USA. ${ }^{19}$ University of Arkansas, Fayetteville, AR, USA. ${ }^{20}$ Mayo Clinic, Rochester, MN, USA. ${ }^{21}$ UC Davis, Davis, CA, USA. ${ }^{22}$ Mt. Sinai, New York, NY, USA. ${ }^{23}$ Helmholtz Zentrum München, Munich, Germany. ${ }^{24}$ Max Planck, Munich, Germany. ${ }^{25}$ University of Oxford, Oxford, UK. ${ }^{26}$ Emory Univ, Atlanta, GA, USA. ${ }^{27}$ Amsterdam University, Amsterdam, Netherlands. ${ }^{28}$ Rush Univ, Chicago, IL, USA
} 